To appear in International Journal of Theoretical and Applied Finance (15 pp)

\title{
Defaultable claims in switching models with partial information
}

\author{
Pavel V. Gapeev* $\quad$ Monique Jeanblanc ${ }^{\dagger}$
}

We study a credit risk model for a financial market in which the local drift rate of the logarithm of the intensity of the default time changes at the times at which certain unobservable external events occur. The risk-neutral dynamics of the default intensity are described by a generalised geometric Brownian motion and the changes of the local drift rate arrive at independent exponential times. We obtain closed form expressions for the rational values of defaultable European-style contingent claims through the filtering estimates of the occurrence of switching times given the filtration generated by the default intensity process.

\section{Introduction}

In this paper, we consider a credit risk model in which the drift rate of the default intensity process changes its form at some unobservable random times. Such a model is related to a financial market in which the occurrence of some external events leads to structural changes in the default intensity rate under the risk-neutral probability measure. For instance, such a situation may happen when the failure of a large industrial company or some important political decision taken by the government can affect the default policy of the issuing firm. Suppose that the dynamics of the logarithm of the intensity rate of the default time are described by a generalised geometric Brownian motion with random drift rates changing their values in some exponentially distributed random times which are independent of each other and of the driving standard Brownian motion. We derive closed form expressions for the rational (no-arbitrage) values of some European-type contingent claims in the model of financial market described above. It is assumed that the payoff of the contingent claims are (measurable) functions of the default intensity value at the maturity time. Note that the consideration of defaultable

*London School of Economics, Department of Mathematics, Houghton Street, London WC2A 2AE, United Kingdom; e-mail: p.v.gapeev@lse.ac.uk

†Univ Evry-Université Paris Saclay, LaMME, 23 Boulevard de France, 91037 Evry Cedex; e-mail: monique.jeanblanc@univ-evry.fr

Mathematics Subject Classification 2000: Primary 91B70, 60J60, 62M20. Secondary 91B28, 60J65, 60J25.

Key words and phrases: Contingent claims, geometric Brownian motion, random drift rate, switching time, partial information, filtering equations, posterior probabilities, conditional probability density.

Date: February 11, 2019 
contingent claims with constant payoffs appears of the same difficulty as of the general ones studied in the paper.

The present paper continues the study of the information-based approach for derivative pricing which was initiated by Brody, Hughston, and Macrina [2] for the case of a multidimensional diffusion model with switching random drifts. The paper can also be considered as a continuation of our research presented in [6]-[7] where we studied the problem of pricing of European-type contingent claims in two-dimensional models with random and switching dividends. The hidden Markov model with finitely many random changes in the drift rate of the default intensity process can be considered as an extension of the model with a single change in the drift rate of observable Brownian motion introduced by Shiryaev [13] (see also [14; Chapter IV, Section 4] and [15; Chapter IV, Section 4]) with the aim to provide a sequential procedure of detecting an unobservable switching (disorder) time. Models with more complicated hidden continuous-time Markov chains as unobservable signals were studied in the literature and the corresponding finite-dimensional systems of Markovian filtering estimates were derived (see, e.g. Liptser and Shiryaev [10; Chapter IX] or Elliott, Aggoun, and Moore [5] for further developments). The analysis of such models represents an important part of general stochastic filtering theory (see, e.g. Kallianpur [9] for an extensive overview).

In this paper, we also propose a simple derivation of the multi-dimensional Markovian system of stochastic differential equations for the posterior probability processes which are filtering estimates of the occurrence of the switching times driven by an innovation standard Brownian motion. Since the marginal distribution of the multi-dimensional Markov process formed by the default intensities together with the resulting posterior probabilities certainly has a complicated structure, our main tool for the derivation of the pricing formulas for the contingent claims is based on the application of the so-called key lemma of credit risk theory. The rational values of the contingent claims are thus expressed through the joint transition density of an exponential Brownian motion and the associated Lebesgue integral derived by Yor [16].

The paper is organised as follows. In Section 2, we introduce a model with the default intensity based on a generalised geometric Brownian motion with switching local drift rates described above. In Section 3, we derive stochastic differential equations for the posterior probabilities of the times of occurrence of external events and get explicit expressions for their conditional probability density given the accessible filtration generated by the default intensity rate. In Section 4, we obtain closed form expressions for rational prices of European-type contingent claims under partial information generated by the default intensity process. The main results of the paper are stated in Propositions 3.1 and 4.1.

\section{The model}

In this section, we introduce a model of a financial market containing a default time which has the intensity process modeled by a generalised exponential Brownian motion, with a switching drift rate. 


\subsection{The dynamics of default intensity}

Let us suppose that on a probability space $(\Omega, \mathcal{G}, P)$ there exists a standard Brownian motion $W=\left(W_{t}\right)_{t \geq 0}$ and independent non-negative random variables $\zeta_{k}, k=1, \ldots, n, n \in \mathbb{N}$, as well as a random variable $U$ which is uniformly distributed on $(0,1)$ and independent of the process $W$ and of the variables $\zeta_{k}, k=1, \ldots, n$. Assume for simplicity that the process $W$ and the variables $\zeta_{k}, k=1, \ldots, n$, are independent and the latter have exponential distribution $P\left(\zeta_{k}>t\right)=e^{-\lambda t}$, for all $t \geq 0$ and some $\lambda>0$. We also define a non-decreasing finite sequence of random variables $\left(\theta_{k}\right)_{k=1, \ldots, n}$ by $\theta_{k}=\zeta_{1}+\zeta_{2}+\cdots+\zeta_{k}, k=1, \ldots, n$. Define the random time $\tau$ by

$$
\tau=\inf \left\{t \geq 0 \mid A_{t} \geq-\ln U\right\},
$$

where the process $A=\left(A_{t}\right)_{t \geq 0}$ is given by

$$
A_{t}=\int_{0}^{t} Y_{s} d s
$$

for all $t \geq 0$, so that $A$ is the cumulative intensity of the random time $\tau$. Assume that the intensity rate process $Y=\left(Y_{t}\right)_{t \geq 0}$ admits the representation

$$
Y_{t}=\exp \left(\left(\beta_{0}-\frac{\sigma^{2}}{2}\right) t+\sum_{k=1}^{n}\left(\beta_{k}-\beta_{k-1}\right)\left(t-\theta_{k}\right)^{+}+\sigma W_{t}\right)
$$

and thus, solves the stochastic differential equation

$$
d Y_{t}=\left(\beta_{0}+\sum_{k=1}^{n}\left(\beta_{k}-\beta_{k-1}\right) I\left(\theta_{k} \leq t\right)\right) Y_{t} d t+\sigma Y_{t} d W_{t},
$$

where $\left(t-\theta_{k}\right)^{+}=\max \left\{t-\theta_{k}, 0\right\}$, and $I(\cdot)$ denotes the indicator function, while $\beta_{k} \neq \beta_{k-1}$, $k=1, \ldots, n$, and $\sigma>0$ are some given constants.

Suppose that $\tau$ represents the default time of a firm (reference credit) and the process $Y=\left(Y_{t}\right)_{t \geq 0}$ describes the risk-neutral dynamics of the default intensity rate. We assume that the drift rate of the process $\ln Y$ changes at the times $\theta_{k}, k=1, \ldots, n$, at which some systemic changes (external events) occur. For instance, such a behavior of the default intensity rate can be related to the the changes of the economic state of the firm. The purpose of the present paper is to determine the rational prices of some defaultable European contingent claims with a fixed time horizon $T>0$.

\subsection{The rational prices of defaultable European contingent claims}

In what follows, we determine the rational (no-arbitrage) prices of European-style contingent claims with payoffs of the form $C\left(Y_{T}\right)$, for some non-negative measurable functions $C(y)$, $y>0$, and a fixed time horizon $T>0$. We assume that the information available from the market is generated by the default intensity rate $Y$ only, that is related to a situation where the small investors trading in the market cannot observe the times $\theta_{k}, k=1, \ldots, n$, at which the systemic changes (external events) occur. It follows from the so-called key lemma (see, e.g. 
[3; page 122] or [1; Section 5.1]) that the rational (no-arbitrage) price process $V=\left(V_{t}\right)_{t \geq 0}$ of such a claim admits the representation

$$
V_{t} \equiv E\left[e^{-r(T-t)} C\left(Y_{T}\right) I(T<\tau) \mid \mathcal{G}_{t}\right]=I(t<\tau) E\left[e^{-r(T-t)-\left(A_{T}-A_{t}\right)} C\left(Y_{T}\right) \mid \mathcal{F}_{t}\right]
$$

for $0 \leq t \leq T$, where $r \geq 0$ is the interest rate of a riskless bank account and the expectation is taken with respect to the pricing measure under which the dynamics of $Y$ are given by (2.3)-(2.4). Here, we define the progressively enlarged filtration $\left(\mathcal{G}_{t}\right)_{t \geq 0}$ by $\mathcal{G}_{t}=\mathcal{F}_{t} \vee \sigma(\tau \wedge t)$, where $\left(\mathcal{F}_{t}\right)_{t \geq 0}$ is the natural filtration of the process $Y$ given by $\mathcal{F}_{t}=\sigma\left(Y_{s} \mid 0 \leq s \leq t\right)$, for all $t \geq 0$. It is further assumed that all the considered filtrations are regularised to be rightcontinuous and completed by all the sets of $P$-measure zero, so that we actually consider in fact the smallest right continuous filtration that contains $\left(\mathcal{F}_{t} \vee \sigma(\tau \wedge t)\right)_{t \geq 0}$. The filtration $\left(\mathcal{F}_{t}\right)_{t \geq 0}$ reflects the information flow which is accessible for investors trading in the market, while the filtration $\left(\mathcal{G}_{t}\right)_{t \geq 0}$ reflects the accessible information progressively enlarged by the default time $\tau$.

For simplicity of presentation, in the fourth section of the paper, we shall restrict our attention to the case of two subsequent systemic changes (external events) $n=2$. In this case, the value of (2.5) can be decomposed as

$$
\begin{aligned}
V_{t}= & I(t<\tau) E\left[e^{-r(T-t)-\left(A_{T}-A_{t}\right)} C\left(Y_{T}\right) I\left(T<\theta_{1}\right) \mid \mathcal{F}_{t}\right] \\
& +I(t<\tau) E\left[e^{-r(T-t)-\left(A_{T}-A_{t}\right)} C\left(Y_{T}\right) I\left(\theta_{1} \leq T<\theta_{2}\right) \mid \mathcal{F}_{t}\right] \\
& +I(t<\tau) E\left[e^{-r(T-t)-\left(A_{T}-A_{t}\right)} C\left(Y_{T}\right) I\left(\theta_{1}<\theta_{2} \leq T\right) \mid \mathcal{F}_{t}\right]
\end{aligned}
$$

for all $0 \leq t \leq T$. The case of general $n \in \mathbb{N}$ can be obtained using the same methodology but with longer computations.

\subsection{Some filtrations and distribution laws}

In order to compute the conditional expectations in (2.6), let us define the processes $Y^{k}=$ $\left(Y_{t}^{k}\right)_{t \geq 0}$ and $A^{k}=\left(A_{t}^{k}\right)_{t \geq 0}$ by

$$
Y_{t}^{k}=\exp \left(\left(\beta_{k}-\frac{\sigma^{2}}{2}\right) t+\sigma W_{t}\right) \quad \text { and } \quad A_{t}^{k}=\int_{0}^{t} Y_{s}^{k} d s
$$

for every $k=0,1, \ldots, n$. Let us now introduce the progressively enlarged filtrations $\left(\mathcal{G}_{t}^{k}\right)_{t \geq 0}$ by $\mathcal{G}_{t}^{k-1} \vee \sigma\left(\theta_{k} \wedge t\right)$, for all $t \geq 0$, so that $\theta_{k}$ is a $\left(\mathcal{G}_{t}^{k}\right)_{t>0}$-stopping time, for every $k=1, \ldots, n$. Moreover, we define the initially enlarged filtration $\left(\mathcal{F}_{t}^{k}\right)_{t \geq 0}$ by $\mathcal{F}_{t}^{k-1} \vee \sigma\left(\theta_{k}\right)$, for all $t \geq 0$ and every $k=1, \ldots, n$, where we set $\mathcal{G}_{t}^{0}=\mathcal{F}_{t}^{0}=\sigma\left(W_{s} \mid 0 \leq s \leq t\right)$, for $t \geq 0$. Observe that the inclusion $\mathcal{F}_{t} \subseteq \mathcal{G}_{t}^{n}$ holds, for all $t \geq 0$, by construction.

Let us now consider a filtration $\left(\mathcal{K}_{t}\right)_{t \geq 0}$ larger than the filtration $\left(\mathcal{F}_{t}\right)_{t \geq 0}$, that is, $\mathcal{F}_{t} \subseteq \mathcal{K}_{t}$, for all $t \geq 0$. Then, if $\mathcal{K}_{t}$ coincides with $\mathcal{F}_{t}$ on the event $J_{t} \in \mathcal{K}_{t}$, that is, if for any $K_{t} \in \mathcal{K}_{t}$, there exists an event $F_{t} \in \mathcal{F}_{t}$ such that $J_{t} \cap K_{t}=J_{t} \cap F_{t}$, then the random variable $E\left[Z_{T} \mid \mathcal{K}_{t}\right]$, on the set $J_{t}$, is equal to an $\mathcal{F}_{t}$-measurable random variable, which, as in [3; page 122] or [1; Section 5.1], leads to the equality

$$
I\left(J_{t}\right) E\left[Z_{T} \mid \mathcal{K}_{t}\right] P\left(J_{t} \mid \mathcal{F}_{t}\right)=I\left(J_{t}\right) E\left[Z_{T} I\left(J_{t}\right) \mid \mathcal{F}_{t}\right]
$$


for each $\mathcal{K}_{T}$-measurable (positive) integrable random variable $Z_{T}$. In this case, when $P\left(J_{t} \mid \mathcal{F}_{t}\right)$ is positive on $J_{t}$, we have

$$
I\left(J_{t}\right) E\left[Z_{T} \mid \mathcal{K}_{t}\right]=I\left(J_{t}\right) \frac{E\left[Z_{T} I\left(J_{t}\right) \mid \mathcal{F}_{t}\right]}{P\left(J_{t} \mid \mathcal{F}_{t}\right)}
$$

for each $\mathcal{K}_{T}$-measurable integrable random variable $Z_{T}$. We further refer to the result in (2.8)-(2.9) as to the generalised key lemma.

As it follows from the results below, the process $(Y, A)$ defined in $(2.2)$ and $(2.3)$ has a complicated Markovian structure on its natural filtration $\left(\mathcal{F}_{t}\right)_{t \geq 0}$, so that, the direct computation of the conditional expectations in (2.5) should be avoided. Therefore, taking into account the tower property for conditional expectations, we obtain from the expressions in (2.3)-(2.4) that, in the case $n=2$, the value process from (2.5) and (2.6) admits the representation

$$
\begin{aligned}
V_{t}= & I(t<\tau) E\left[E\left[e^{-r(T-t)-\left(A_{T}-A_{t}\right)} C\left(Y_{T}\right) I\left(T<\theta_{1}\right) \mid \mathcal{G}_{t}^{2}\right] \mid \mathcal{F}_{t}\right] \\
& +I(t<\tau) E\left[E\left[e^{-r(T-t)-\left(A_{T}-A_{t}\right)} C\left(Y_{T}\right) I\left(\theta_{1} \leq T<\theta_{2}\right) \mid \mathcal{G}_{t}^{2}\right] \mid \mathcal{F}_{t}\right] \\
& +I(t<\tau) E\left[E\left[e^{-r(T-t)-\left(A_{T}-A_{t}\right)} C\left(Y_{T}\right) I\left(\theta_{1}<\theta_{2} \leq T\right) \mid \mathcal{G}_{t}^{2}\right] \mid \mathcal{F}_{t}\right]
\end{aligned}
$$

for all $0 \leq t \leq T$.

Let us now refer the explicit expression for the transition density function of the processes $\left(Y^{k}, A^{k}\right), k=0,1, \ldots, n$, defined in (2.7) above. For this purpose, we recall from [16; page 527] that the random variable $A_{t}^{(\nu)}=\int_{0}^{t} e^{2\left(W_{s}+\nu s\right)} d s$ has the conditional distribution

$$
P\left(A_{t}^{(\nu)} \in d a \mid W_{t}+\nu t=x\right)=p(t, x, a) d a
$$

where the density function $p$ is given by:

$$
\begin{aligned}
p(t, x, a)= & \frac{1}{\pi a^{2}} \exp \left(\frac{x^{2}+\pi^{2}}{2 t}+x-\frac{1+e^{2 x}}{2 a}\right) \\
& \times \int_{0}^{\infty} \exp \left(-\frac{w^{2}}{2 t}-\frac{e^{x}}{a} \cosh (w)\right) \sinh (w) \sin \left(\frac{\pi w}{t}\right) d w
\end{aligned}
$$

with $t, a>0$ and $x \in \mathbb{R}$, and $\nu \in \mathbb{R}$ given and fixed. This fact yields that the random vector $\left(2\left(W_{t}+\nu t\right), A_{t}^{(\nu)}\right)$ has the distribution

$$
P\left(2\left(W_{t}+\nu t\right) \in d x, A_{t}^{(\nu)} \in d a\right)=q(t, x, a) d x d a,
$$

where the density function $q$ is given by

$$
\begin{aligned}
q(t, x, a)= & p\left(t, \frac{x}{2}, a\right) \frac{1}{2 \sqrt{t}} \frac{1}{\sqrt{2 \pi}} \exp \left(-\frac{1}{2}\left(\frac{x-2 \nu t}{2 \sqrt{t}}\right)^{2}\right) \\
= & \frac{1}{(2 \pi)^{3 / 2} a^{2} \sqrt{t}} \exp \left(\frac{\pi^{2}}{2 t}+\left(\frac{\nu+1}{2}\right) x-\frac{\nu^{2}}{2} t-\frac{1+e^{x}}{2 a}\right) \\
& \times \int_{0}^{\infty} \exp \left(-\frac{w^{2}}{2 t}-\frac{e^{x / 2}}{a} \cosh (w)\right) \sinh (w) \sin \left(\frac{\pi w}{t}\right) d w
\end{aligned}
$$


with $t, a>0$ and $x \in \mathbb{R}$ (see also [4] and [12] for related expressions in terms of Hermite functions). Therefore, using the fact that the scaling property of $W$ implies

$$
\begin{aligned}
& P\left(\left(\beta_{k}-\frac{\sigma^{2}}{2}\right) t+\sigma W_{t} \leq x, \int_{0}^{t} e^{\left(\beta_{k}-\sigma^{2} / 2\right) s+\sigma W_{s}} d s \leq a\right) \\
& =P\left(2\left(W_{t^{\prime}}+\nu t^{\prime}\right) \leq x, \int_{0}^{t^{\prime}} e^{2\left(W_{s}+\nu s\right)} d s \leq \frac{\sigma^{2} a}{4}\right)
\end{aligned}
$$

with $t^{\prime}=\sigma^{2} t / 4$ and $\nu=2 \beta_{k} / \sigma^{2}-1$, by virtue of the expressions in (2.13)-(2.14), it follows from the definition in (2.7) and the Markov property of the process $\left(Y^{k}, A^{k}\right), k=0,1, \ldots, n$, that the random vector $\left(Y_{T}^{k} / Y_{t}^{k},\left(A_{T}^{k}-A_{t}^{k}\right) / Y_{t}^{k}\right)$ has the distribution

$$
P\left(Y_{T}^{k} / Y_{t}^{k} \in d y,\left(A_{T}^{k}-A_{t}^{k}\right) / Y_{t}^{k} \in d a\right)=P\left(Y_{T-t}^{k} \in d y, A_{T-t}^{k} \in d a\right)=g_{k}(T-t, y, a) d y d a,
$$

where the density function $g_{k}$ is given by

$$
\begin{aligned}
& g_{k}(T-t, y, a)=\frac{\sigma^{2}}{4 y} q\left(\frac{\sigma^{2}}{4}(T-t), \ln (y), \frac{\sigma^{2} a}{4}\right) \\
& =\frac{2 \sqrt{2}}{\pi^{3 / 2} \sigma^{3}} \frac{1}{a^{2} y \sqrt{T-t}} \exp \left(\frac{2 \pi^{2}}{\sigma^{2}(T-t)}+\frac{\beta_{k}}{\sigma^{2}} \ln (y)-\frac{\left(\beta_{k} / \sigma-\sigma / 2\right)^{2}}{2}(T-t)-\frac{2(1+y)}{\sigma^{2} a}\right) \\
& \quad \times \int_{0}^{\infty} \exp \left(-\frac{2 w^{2}}{\sigma^{2}(T-t)}-\frac{4 \sqrt{y}}{\sigma^{2} a} \cosh (w)\right) \sinh (w) \sin \left(\frac{4 \pi w}{\sigma^{2}(T-t)}\right) d w
\end{aligned}
$$

for all $T-t, y, a>0$. Note that the formulas above were used in [8] for the computation of the marginal density of the posterior probability process $\Pi^{1}$ introduced below.

\section{$3 \quad$ Filtering equations and conditional densities}

In this section, we derive stochastic differential equations for the posterior probabilities of occurrence of external events and their conditional probability density with respect to the accessible filtration $\left(\mathcal{F}_{t}\right)_{t \geq 0}$.

\subsection{Posterior probabilities}

In our model, the distribution of the process $\ln Y$ with respect to the probability measure $P$ has the structure

$$
P(\ln Y \in \cdot)=\int_{0}^{\infty} \int_{s_{1}}^{\infty} \cdots \int_{s_{n-1}}^{\infty} P^{s_{1}, s_{2}, \ldots, s_{n}}(\ln Y \in \cdot) \lambda^{n} e^{-\lambda s_{n}} d s_{n} \cdots d s_{2} d s_{1},
$$

where $P^{s_{1}, s_{2}, \ldots, s_{n}}(\ln Y \in \cdot)=P\left(\ln Y \in \cdot \mid \theta_{1}=s_{1}, \theta_{2}=s_{2}, \ldots, \theta_{n}=s_{n}\right)$ are the distribution laws of the corresponding Brownian motions with diffusion coefficient $\sigma^{2}$ and the drift rate changing from $\beta_{k-1}-\sigma^{2} / 2$ to $\beta_{k}-\sigma^{2} / 2$ at the times $\theta_{k}, k=1, \ldots, n$.

Taking into account the fact that the probability measure $P^{s_{1}, s_{2}, \ldots, s_{n}}$ is equivalent to $P$ on $\mathcal{F}_{t}$ by construction, for any $0 \leq s_{1} \leq s_{2} \leq \ldots \leq s_{n} \leq \infty$, applying the generalised Bayes' formula 
(see, e.g. [10; Theorem 7.23]), we get that the posterior probability processes $\Pi^{k}=\left(\Pi_{t}^{k}\right)_{t \geq 0}$ defined by $\Pi_{t}^{k}=P\left(\theta_{k} \leq t \mid \mathcal{F}_{t}\right)$ take the form

$$
\Pi_{t}^{k}=\left.\int_{0}^{t} \int_{s_{1}}^{t} \cdots \int_{s_{k-1}}^{t} \int_{s_{k}}^{\infty} \cdots \int_{s_{n-1}}^{\infty} \frac{d P^{s_{1}, s_{2}, \ldots, s_{n}}}{d P}\right|_{\mathcal{F}_{t}} \lambda^{n} e^{-\lambda s_{n}} d s_{n} \cdots d s_{2} d s_{1}
$$

for all $t \geq 0$. Moreover, since the measure $P^{s_{1}, s_{2}, \ldots, s_{k}, s_{k+1}, \ldots, s_{n}}$ coincides with $P^{s_{1}, s_{2}, \ldots, s_{k}, t, \ldots, t}$ on $\mathcal{F}_{t}$, for all $0 \leq s_{1} \leq s_{2} \leq \ldots \leq s_{k} \leq t \leq s_{k+1} \leq \ldots \leq s_{n}$, we see that

$$
\begin{aligned}
\Pi_{t}^{k}-\Pi_{t}^{k+1} & =\left.\int_{0}^{t} \int_{s_{1}}^{t} \cdots \int_{s_{k-1}}^{t} \int_{t}^{\infty} \int_{s_{k+1}}^{\infty} \cdots \int_{s_{n-1}}^{\infty} \frac{d P^{s_{1}, s_{2}, \ldots, s_{n}}}{d P}\right|_{\mathcal{F}_{t}} \lambda^{n} e^{-\lambda s_{n}} d s_{n} \cdots d s_{2} d s_{1} \\
& =\left.\int_{0}^{t} \int_{s_{1}}^{t} \cdots \int_{s_{k-1}}^{t} \frac{d P^{s_{1}, s_{2}, \ldots, s_{k}, t, \ldots, t}}{d P}\right|_{\mathcal{F}_{t}} \lambda^{k} e^{-\lambda t} d s_{k} \cdots d s_{2} d s_{1}
\end{aligned}
$$

is satisfied, where the last equality holds, because the probability measure $P^{s_{1}, s_{2}, \ldots, s_{n}}$ coincides with $P^{s_{1}, s_{2}, \ldots, s_{k}, t, \ldots, t}$ on $\mathcal{F}_{t}$, for any $0 \leq s_{1} \leq \ldots \leq s_{k} \leq t \leq s_{k+1} \leq \ldots \leq s_{n}$ fixed. By means of Girsanov's theorem for diffusion processes (see, e.g. [10; Theorem 7.19]), it follows from the structure of the observation process $\ln Y$ that the representations

$$
\left.\frac{d P^{s_{1}, \ldots, s_{k}, s_{k+1}, \ldots, s_{n}}}{d P^{t, \ldots, t, t, \ldots, t}}\right|_{\mathcal{F}_{t}}=\left.\frac{d P^{s_{1}, \ldots, s_{k}, t, \ldots, t}}{d P^{t, \ldots, t, t, \ldots, t}}\right|_{\mathcal{F}_{t}}=\prod_{j=1}^{k} \frac{L_{t}^{j}}{L_{s_{j}}^{j}}
$$

hold for all $0 \leq s_{1} \leq \ldots \leq s_{k} \leq t \leq s_{k+1} \leq \ldots \leq s_{n}$, where the likelihood ratio process $L^{j}=\left(L_{t}^{j}\right)_{t \geq 0}$ has the form

$$
L_{t}^{j}=\exp \left(\frac{\beta_{j}-\beta_{j-1}}{\sigma^{2}} \ln Y_{t}-\frac{\left(\beta_{j}-\sigma^{2} / 2\right)^{2}-\left(\beta_{j-1}-\sigma^{2} / 2\right)^{2}}{2 \sigma^{2}} t\right)
$$

for every $j=1, \ldots, k$, in terms of the logarithm of the asset price process $Y$ given by

$$
\ln Y_{t}=\left(\beta_{0}-\frac{\sigma^{2}}{2}\right) t+\sum_{k=1}^{n}\left(\beta_{k}-\beta_{k-1}\right)\left(t-\theta_{k}\right)^{+}+\sigma W_{t}
$$

for all $t \geq 0$ and every $k=0,1, \ldots, n$.

It is then shown by means of straightforward calculations that the posterior probabilities $\Pi^{k}$ from (3.2) have the form

$$
\Pi_{t}^{1}=\frac{\Xi_{t}^{n}}{1+\Xi_{t}^{n}} \quad \text { and } \quad \Pi_{t}^{k}-\Pi_{t}^{k+1}=\frac{\Phi_{t}^{k}}{1+\Xi_{t}^{n}} \quad \text { with } \quad \Xi_{t}^{n}=\sum_{k=1}^{n} \Phi_{t}^{k},
$$

where the weighted likelihood ratio processes $\Phi^{k}=\left(\Phi_{t}^{k}\right)_{t \geq 0}$ are given by

$$
\begin{aligned}
\Phi_{t}^{k} & =\int_{0}^{t} \int_{s_{1}}^{t} \cdots \int_{s_{k-1}}^{t} \prod_{i=1}^{k} \frac{L_{t}^{i}}{L_{s_{i}}^{i}} \int_{t}^{\infty} \int_{s_{k+1}}^{\infty} \cdots \int_{s_{n-1}}^{\infty} \lambda^{n} e^{\lambda\left(t-s_{n}\right)} d s_{n} \cdots d s_{2} d s_{1} \\
& =\int_{0}^{t} \int_{s_{1}}^{t} \cdots \int_{s_{k-1}}^{t} \prod_{i=1}^{k} \frac{L_{t}^{i}}{L_{s_{i}}^{i}} \lambda^{k} d s_{k} \cdots d s_{2} d s_{1}
\end{aligned}
$$

for $k=1, \ldots, n$. 


\subsection{Filtering equations for the posterior probabilities}

Applying Itô's formula (see, e.g. [10; Chapter IV, Theorem 4.4] or [11; Chapter IV, Theorem 3.3]) to the expression in (3.5), we get that the process $L^{j}$ solves the stochastic differential equation

$$
d L_{t}^{j}=L_{t}^{j} \frac{\beta_{j}-\beta_{j-1}}{\sigma^{2}}\left(d \ln Y_{t}-\left(\beta_{j-1}-\sigma^{2} / 2\right) d t\right)
$$

with $L_{0}^{j}=1$. Then, using straightforward computations, we obtain that the processes $\Phi^{k}$, $k=1, \ldots, n$, satisfy the equations

$$
d \Phi_{t}^{k}=\lambda \Phi_{t}^{k-1} d t+\frac{\beta_{k}-\beta_{k-1}}{\sigma^{2}} \Phi_{t}^{k}\left(d \ln Y_{t}-\left(\beta_{k-1}-\sigma^{2} / 2\right) d t\right)
$$

with $\Phi_{t}^{0} \equiv 1$, for $k=1, \ldots, n$, so that the process $\Xi^{n}$ from (3.7) admits the representation

$$
d \Xi_{t}^{n}=\lambda\left(1+\Xi_{t}^{n}\right) d t+\left(1+\Xi_{t}^{n}\right)\left(\sum_{k=1}^{n} \frac{\beta_{k}-\beta_{k-1}}{\sigma^{2}} \Pi_{t}^{k}\right)\left(d \ln Y_{t}-\left(\beta_{0}-\sigma^{2} / 2\right) d t\right) .
$$

Hence, applying Itô's formula to the expressions in (3.7), we get

$$
\begin{aligned}
d\left(\Pi_{t}^{k}-\Pi_{t}^{k+1}\right)= & \lambda\left(\Pi_{t}^{k-1}-2 \Pi_{t}^{k}+\Pi_{t}^{k+1}\right) d t \\
& +\left(\Pi_{t}^{k}-\Pi_{t}^{k+1}\right)\left(\sum_{i=1}^{k} \frac{\beta_{i}-\beta_{i-1}}{\sigma}\left(1-\Pi_{t}^{i}\right)-\sum_{j=k+1}^{n} \frac{\beta_{j}-\beta_{j-1}}{\sigma} \Pi_{t}^{j}\right) d \bar{W}_{t}
\end{aligned}
$$

for $k=1, \ldots, n$, where we set $\Pi_{t}^{0} \equiv 1$. Thus, the processes $\Pi^{k}$ solve the stochastic differential equations

$$
\begin{aligned}
d \Pi_{t}^{k}= & \lambda\left(\Pi_{t}^{k-1}-\Pi_{t}^{k}\right) d t \\
& +\left(\Pi_{t}^{k} \sum_{j=1}^{k} \frac{\beta_{j}-\beta_{j-1}}{\sigma}\left(1-\Pi_{t}^{j}\right)+\left(1-\Pi_{t}^{k}\right) \sum_{j=k+1}^{n} \frac{\beta_{j}-\beta_{j-1}}{\sigma} \Pi_{t}^{j}\right) d \bar{W}_{t}
\end{aligned}
$$

for $k=1, \ldots, n$. Here, the innovation process $\bar{W}=\left(\bar{W}_{t}\right)_{t \geq 0}$ defined by

$$
\bar{W}_{t}=\frac{1}{\sigma}\left(\ln Y_{t}-\int_{0}^{t}\left(\beta_{0}-\frac{\sigma^{2}}{2}+\sum_{k=1}^{n}\left(\beta_{k}-\beta_{k-1}\right) \Pi_{s}^{k}\right) d s\right)
$$

is a standard Brownian motion under the probability measure $P$, with respect to the filtration $\left(\mathcal{F}_{t}\right)_{t \geq 0}$, according to P. Lévy's characterisation theorem (see, e.g. [10; Theorem 4.1] or [11; Chapter IV, Theorem 3.6]).

\subsection{Conditional densities of the switching times}

Let us now find an expression for the family of conditional probability density processes $\left(\alpha_{t}\left(u_{1}, u_{2}, \ldots, u_{n}\right)\right)_{t \geq 0}$ defined from the expression

$$
\begin{aligned}
& P\left(\theta_{1}>u_{1}, \theta_{2}>u_{2}, \ldots, \theta_{n}>u_{n} \mid \mathcal{F}_{t}\right) \\
& =\int_{u_{1}}^{\infty} \int_{u_{2}}^{\infty} \ldots \int_{u_{n}}^{\infty} \alpha_{t}\left(v_{1}, v_{2}, \ldots, v_{n}\right) I\left(v_{1}<v_{2}<\ldots<v_{n}\right) \lambda^{n} e^{-\lambda v_{n}} d v_{1} d v_{2} \ldots d v_{n}
\end{aligned}
$$


for all $u_{1}<u_{2}<\ldots<u_{n}$ and $t \geq 0$. It follows from the straightforward computations above that the the processes $\left(\alpha_{t}\left(u_{1}, u_{2}, \ldots, u_{n}\right)\right)_{t \geq 0}$ admit the representations

$$
\alpha_{t}\left(u_{1}, u_{2}, \ldots, u_{n}\right)=\frac{e^{\lambda t}}{1+\Xi_{t}^{n}} \frac{L_{t}^{1}}{L_{u_{1} \wedge t}^{1}} \frac{L_{t}^{2}}{L_{u_{2} \wedge t}^{2}} \cdots \frac{L_{t}^{n}}{L_{u_{n} \wedge t}^{n}}
$$

for all $u_{1}<u_{2}<\ldots<u_{n}$ and $t \geq 0$. Here, the processes $L^{j}, j=1, \ldots, n$, and $\Xi^{n}$ are defined in (3.5) and (3.7) above. Furthermore, taking into account the structure of the processes $\Pi^{k}$ and $\Phi^{k}, k=1, \ldots, n$, in (3.2) and (3.8), by means of standard arguments, we can verify that

$$
\int_{0}^{\infty} \int_{0}^{\infty} \cdots \int_{0}^{\infty} \alpha_{t}\left(u_{1}, u_{2}, \ldots, u_{n}\right) I\left(u_{1}<u_{2}<\ldots<u_{n}\right) \lambda^{n} e^{-\lambda u_{n}} d u_{1} d u_{2} \cdots u_{n}=1
$$

as expected. This shows the regularity of the family of conditional probability density processes $\left(\alpha_{t}\left(u_{1}, u_{2}, \ldots, u_{n}\right)\right)_{t \geq 0}$, for any $u_{1}<u_{2}<\ldots<u_{n}$ fixed.

Summarising the facts proved above, let us formulate the following assertion.

Proposition 3.1. In the model for $Y$ of (2.3)-(2.4) with partial information contained in $\left(\mathcal{F}_{t}\right)_{t \geq 0}$, the posterior probability $\left(\Pi^{1}, \Pi^{2}, \ldots, \Pi^{n}\right)$ from $(3.7)$ is an $n$-dimensional while $\left(Y, \Pi^{1}, \Pi^{2}, \ldots, \Pi^{n}\right)$ forms an $n+1$-dimensional time-homogeneous Markov process. Moreover, the conditional probability density $\alpha_{t}\left(u_{1}, u_{2}, \ldots, u_{n}\right)$ defined in (3.15) admits the representation of (3.16), for any $u_{1}<u_{2}<\ldots<u_{n}$ fixed, where the processes $L^{j}, j=1, \ldots, n$, and $\Xi^{n}$ are given by (3.5) and (3.7), respectively.

\section{Computation of the rational prices}

In this section, we compute the three conditional expectations of the expressions in (2.6) and (2.10) corresponding to the case $n=2$. The computation of the appropriate terms corresponding to the case of general $n \in \mathbb{N}$ can be obtained using the same methodology but with longer computations. To simplify the notations, without loss of generality, we further assume that the payoffs are already discounted by the dynamics of the bank account, that is equivalent to letting the interest rate $r$ equal to zero.

\subsection{The first term}

Let us begin by computing the first term in (2.6). For this purpose, we first observe that the equalities $Y_{T}=Y_{T}^{0}$ and $A_{T}-A_{t}=A_{T}^{0}-A_{t}^{0}$ hold on the event $\left\{T<\theta_{1}\right\}$ and note that $\mathcal{G}_{t}^{2}$ coincides with $\mathcal{G}_{t}^{1}$ on the event $\left\{t<\theta_{1}\right\}$, and thus, the generalised key lemma in (2.8)-(2.9) yields that

$$
E\left[e^{-\left(A_{T}-A_{t}\right)} C\left(Y_{T}\right) I\left(T<\theta_{1}\right) \mid \mathcal{G}_{t}^{2}\right]=I\left(t<\theta_{1}\right) E\left[e^{-\left(A_{T}^{0}-A_{t}^{0}\right)} C\left(Y_{T}^{0}\right) I\left(T<\theta_{1}\right) \mid \mathcal{G}_{t}^{1}\right]
$$

holds, for all $0 \leq t \leq T$, where we have used the fact that $P\left(t<\theta_{1} \mid \mathcal{G}_{t}^{1}\right)=I\left(t<\theta_{1}\right)$. Then, using the fact that $\left(\mathcal{G}_{t}^{1}\right)_{t \geq 0}$ and $\left(\mathcal{F}_{t}^{0}\right)_{t \geq 0}$ coincide on $\left\{t<\theta_{1}\right\}$ and that the probability 
$P\left(t<\theta_{1} \mid \mathcal{F}_{t}^{0}\right)$ is positive on that event, taking into account the independence of the exponential random variable $\zeta_{1}$ and the process $Y^{0}$, we get

$$
\begin{aligned}
& E\left[e^{-\left(A_{T}^{0}-A_{t}^{0}\right)} C\left(Y_{T}^{0}\right) I\left(T<\theta_{1}\right) \mid \mathcal{G}_{t}^{1}\right]=I\left(t<\theta_{1}\right) \frac{E\left[e^{-Y_{t}^{0}\left(A_{T}^{0}-A_{t}^{0}\right) / Y_{t}^{0}} C\left(Y_{T}^{0}\right) I\left(T<\theta_{1}\right) \mid \mathcal{F}_{t}^{0}\right]}{P\left(t<\theta_{1} \mid \mathcal{F}_{t}^{0}\right)} \\
& =I\left(t<\theta_{1}\right) C_{0}\left(T-t, Y_{t}^{0}\right)=I\left(t<\theta_{1}\right) C_{0}\left(T-t, Y_{t}\right)
\end{aligned}
$$

for $0 \leq t \leq T$. Here, by virtue of the independence of $\zeta_{1} \equiv \theta_{1}$ and $Y^{0}$, as well as the Markov property of the process $\left(Y^{0}, A^{0}\right)$, and the fact that the random variable $Y_{s}^{0} / Y_{t}^{0}$ has the same law as $Y_{s-t}^{0}$, for each $0 \leq t \leq s$ fixed, we have

$$
\begin{aligned}
& C_{0}(T-t, y)=E\left[e^{-y A_{T-t}^{0}} C\left(y Y_{T-t}^{0}\right)\right] P\left(T<\zeta_{1}\right) / P\left(t<\zeta_{1}\right) \\
& =e^{-\lambda(T-t)} \int_{0}^{\infty} \int_{0}^{\infty} e^{-y a} C(y z) g_{0}(T-t, z, a) d z d a
\end{aligned}
$$

and the function $g_{0}$ is given in (2.17) above. Hence, by means of the tower property for conditional expectations, using the fact that the arguments from the previous section yield $P\left(t<\theta_{1} \mid \mathcal{F}_{t}\right)=1-\Pi_{t}^{1}$, we obtain from (4.1) and (4.2) that

$$
E\left[e^{-\left(A_{T}-A_{t}\right)} C\left(Y_{T}\right) I\left(T<\theta_{1}\right) \mid \mathcal{F}_{t}\right]=P\left(t<\theta_{1} \mid \mathcal{F}_{t}\right) C_{0}\left(T-t, Y_{t}\right)=\left(1-\Pi_{t}^{1}\right) C_{0}\left(T-t, Y_{t}\right)
$$

holds, for all $0 \leq t \leq T$, where the function $C_{0}(T-t, y)$ is given by (4.3) above.

\subsection{The second term}

Let us continue with computing the second term in (2.6). For this purpose, we observe that the equalities $Y_{s} / Y_{t}=Y_{s}^{1} / Y_{t}^{1}$ for $t \leq s \leq T$ and $A_{T}-A_{t}=Y_{t}\left(A_{T}^{1}-A_{t}^{1}\right) / Y_{t}^{1}$ hold on the event $\left\{\theta_{1} \leq t \leq T<\theta_{2}\right\}$, while the equality $Y_{t}\left(Y_{\theta_{1}} / Y_{t}\right)\left(Y_{s} / Y_{\theta_{1}}\right)=Y_{t}^{0}\left(Y_{\theta_{1}}^{0} / Y_{t}^{0}\right)\left(Y_{s}^{1} / Y_{\theta_{1}}^{1}\right)$ holds on the event $\left\{t<\theta_{1} \leq s<\theta_{2}\right\}$ for $s \leq T$ and $A_{T}-A_{\theta_{1}}+A_{\theta_{1}}-A_{t}=Y_{\theta_{1}}^{0}\left(A_{T}^{1}-A_{\theta_{1}}^{1}\right) / Y_{\theta_{1}}^{1}+A_{\theta_{1}}^{0}-A_{t}^{0}$ holds on $\left\{t<\theta_{1} \leq T<\theta_{2}\right\}$, so that

$$
\begin{aligned}
& E\left[e^{-\left(A_{T}-A_{t}\right)} C\left(Y_{T}\right) I\left(\theta_{1} \leq T<\theta_{2}\right) \mid \mathcal{G}_{t}^{2}\right] \\
& =E\left[e^{-\left(A_{T}-A_{t}\right)} C\left(Y_{t}\left(Y_{T} / Y_{t}\right)\right) I\left(\theta_{1} \leq t<T<\theta_{2}\right) \mid \mathcal{G}_{t}^{2}\right] \\
& \quad+E\left[e^{-\left(A_{T}-A_{\theta_{1}}\right)-\left(A_{\theta_{1}}-A_{t}\right)} C\left(Y_{t}\left(Y_{\theta_{1}} / Y_{t}\right)\left(Y_{T} / Y_{\theta_{1}}\right)\right) I\left(t<\theta_{1} \leq T<\theta_{2}\right) \mid \mathcal{G}_{t}^{2}\right] \\
& \quad=I\left(\theta_{1} \leq t<\theta_{2}\right) E\left[e^{-Y_{t}\left(A_{T}^{1}-A_{t}^{1}\right) / Y_{t}^{1}} C\left(Y_{t}\left(Y_{T}^{1} / Y_{t}^{1}\right)\right) I\left(T<\theta_{2}\right) \mid \mathcal{G}_{t}^{2}\right] \\
& \quad+I\left(t<\theta_{1}\right) E\left[e^{-Y_{\theta_{1}}^{0}\left(A_{T}^{1}-A_{\theta_{1}}^{1}\right) / Y_{\theta_{1}}^{1}-\left(A_{\theta_{1}}^{0}-A_{t}^{0}\right)} C\left(Y_{t}^{0}\left(Y_{\theta_{1}}^{0} / Y_{t}^{0}\right)\left(Y_{T}^{1} / Y_{\theta_{1}}^{1}\right)\right) I\left(t<\theta_{1} \leq T<\theta_{2}\right) \mid \mathcal{G}_{t}^{2}\right]
\end{aligned}
$$

holds, for all $0 \leq t \leq T$. Observe that $\mathcal{G}_{t}^{2}$ coincides with $\mathcal{F}_{t}^{1}$ on the event $\left\{\theta_{1} \leq t<\theta_{2}\right\}$, and thus, we can apply the generalised key lemma for the filtrations $\left(\mathcal{G}_{t}^{2}\right)_{t \geq 0}$ and $\left(\mathcal{F}_{t}^{1}\right)_{t \geq 0}$ on $\left\{\theta_{1} \leq t<\theta_{2}\right\}$ to obtain

$$
\begin{aligned}
& I\left(\theta_{1} \leq t<\theta_{2}\right) E\left[e^{-Y_{t}\left(A_{T}^{1}-A_{t}^{1}\right) / Y_{t}^{1}} C\left(Y_{t}\left(Y_{T}^{1} / Y_{t}^{1}\right)\right) I\left(T<\theta_{2}\right) \mid \mathcal{G}_{t}^{2}\right] \\
& =\frac{I\left(\theta_{1} \leq t<\theta_{2}\right)}{P\left(\theta_{1} \leq t<\theta_{2} \mid \mathcal{F}_{t}^{1}\right)} E\left[e^{-Y_{t}\left(A_{T}^{1}-A_{t}^{1}\right) / Y_{t}^{1}} C\left(Y_{t}\left(Y_{T}^{1} / Y_{t}^{1}\right)\right) I\left(T<\theta_{2}\right) \mid \mathcal{F}_{t}^{1}\right] \\
& =I\left(\theta_{1} \leq t<\theta_{2}\right) C_{1}^{0}\left(T-t, Y_{t}\right)
\end{aligned}
$$


for $0 \leq t \leq T$. Here, using the fact that the $\mathcal{F}_{t}^{1}$-measurable random variable $Y_{t}$ is independent of $Y_{T}^{1} / Y_{t}^{1}$ on the event $\left\{\theta_{1} \leq t \leq T<\theta_{2}\right\}$, by virtue of the independence of the exponential random variables $\zeta_{1} \equiv \theta_{1}, \zeta_{2} \equiv \theta_{2}-\theta_{1}$, and the process $Y^{1}$, as well as the Markov property of the process $\left(Y^{1}, A^{1}\right)$, and the fact that the random variable $Y_{s}^{1} / Y_{t}^{1}$ has the same law as $Y_{s-t}^{1}$, for each $0 \leq t \leq s$ fixed, we have

$$
\begin{aligned}
& C_{1}^{0}(T-t, y)=E\left[e^{-y A_{T-t}^{1}} C\left(y Y_{T-t}^{1}\right)\right] P\left(T<\zeta_{1}+\zeta_{2} \mid \zeta_{1}\right) / P\left(t<\zeta_{1}+\zeta_{2} \mid \zeta_{1}\right) \\
& =e^{-\lambda(T-t)} \int_{0}^{\infty} \int_{0}^{\infty} e^{-y a} C(y z) g_{1}(T-t, z, a) d z d a
\end{aligned}
$$

for $0 \leq t \leq T$, and the function $g_{1}$ is given in (2.17) above. Note that the expression in (4.7) does not depend on $\zeta_{1}$, because of the exponential distribution of the random variable $\zeta_{2}$ and the independence between $\zeta_{1}$ and $\zeta_{2}$. Hence, by means of the tower property for conditional expectations and the fact that the arguments from the previous section yield $P\left(\theta_{1} \leq t<\right.$ $\left.\theta_{2} \mid \mathcal{F}_{t}\right)=\Pi_{t}^{1}-\Pi_{t}^{2}$, we obtain from (4.5) and (4.6) that

$$
\begin{aligned}
& E\left[e^{-\left(A_{T}-A_{t}\right)} C\left(Y_{T}\right) I\left(\theta_{1} \leq t<T<\theta_{2}\right) \mid \mathcal{F}_{t}\right] \\
& =P\left(\theta_{1} \leq t<\theta_{2} \mid \mathcal{F}_{t}\right) C_{1}^{0}\left(T-t, Y_{t}\right)=\left(\Pi_{t}^{1}-\Pi_{t}^{2}\right) C_{1}^{0}\left(T-t, Y_{t}\right)
\end{aligned}
$$

holds, for all $0 \leq t \leq T$, where the function $C_{1}^{0}(T-t, y)$ is given in (4.7) above.

Now, taking into account the fact that $\mathcal{G}_{t}^{2}$ coincides with $\mathcal{F}_{t}^{0}$ on the event $\left\{t<\theta_{1}\right\}$, we can apply the generalised key lemma for the filtrations $\left(\mathcal{G}_{t}^{2}\right)_{t \geq 0}$ and $\left(\mathcal{F}_{t}^{0}\right)_{t \geq 0}$ to get

$$
\begin{aligned}
& I\left(t<\theta_{1}\right) E\left[e^{-Y_{\theta_{1}}^{0}\left(A_{T}^{1}-A_{\theta_{1}}^{1}\right) / Y_{\theta_{1}}^{1}-\left(A_{\theta_{1}}^{0}-A_{t}^{0}\right)} C\left(Y_{t}^{0}\left(Y_{\theta_{1}}^{0} / Y_{t}^{0}\right)\left(Y_{T}^{1} / Y_{\theta_{1}}^{1}\right)\right) I\left(t<\theta_{1} \leq T<\theta_{2}\right) \mid \mathcal{G}_{t}^{2}\right] \\
& =I\left(t<\theta_{1}\right) \frac{E\left[e^{-Y_{\theta_{1}}^{0}\left(A_{T}^{1}-A_{\theta_{1}}^{1}\right) / Y_{\theta_{1}}^{1}-\left(A_{\theta_{1}}^{0}-A_{t}^{0}\right)} C\left(Y_{t}^{0}\left(Y_{\theta_{1}}^{0} / Y_{t}^{0}\right)\left(Y_{T}^{1} / Y_{\theta_{1}}^{1}\right)\right) I\left(t<\theta_{1} \leq T<\theta_{2}\right) \mid \mathcal{F}_{t}^{0}\right]}{P\left(t<\theta_{1} \mid \mathcal{F}_{t}^{0}\right)} \\
& =I\left(t<\theta_{1}\right) C_{1}^{1}\left(T-t, Y_{t}^{0}\right)=I\left(t<\theta_{1}\right) C_{1}^{1}\left(T-t, Y_{t}\right)
\end{aligned}
$$

for all $0 \leq t \leq T$. Here, by virtue of the independence of the exponential random variables $\zeta_{1} \equiv \theta_{1}, \zeta_{2} \equiv \theta_{2}-\theta_{1}$, and $Y^{k}, k=0,1$, as well as the Markov property of the processes $\left(Y^{k}, A^{k}\right), k=0,1$, and the fact that the random variables $Y_{s}^{k} / Y_{t}^{k}$ have the same laws as $Y_{s-t}^{k}$, $k=0,1$, for each $0 \leq t \leq s$, we have

$$
\begin{aligned}
& C_{1}^{1}(T-t, y)=E\left[e^{-y A_{\zeta_{1}-t}^{0}} \widehat{C}_{1}^{1}\left(T-\zeta_{1}, y Y_{\zeta_{1}-t}^{0}\right) I\left(t<\zeta_{1} \leq T\right)\right] / P\left(t<\zeta_{1}\right) \\
& =\int_{t}^{T} \int_{0}^{\infty} \int_{0}^{\infty} e^{-y a} \widehat{C}_{1}^{1}(T-u, y z) \lambda e^{-\lambda(u-t)} g_{0}(u-t, z, a) d u d z d a
\end{aligned}
$$

with

$$
\begin{aligned}
& \widehat{C}_{1}^{1}(T-u, y)=E\left[e^{-y A_{T-u}^{1}} C\left(y Y_{T-u}^{1}\right)\right] P\left(T-u<\zeta_{2}\right) \\
& =e^{-\lambda(T-u)} \int_{0}^{\infty} \int_{0}^{\infty} e^{-y a} C(y z) g_{1}(T-u, z, a) d z d a
\end{aligned}
$$

for $0 \leq t<u \leq T$, and the functions $g_{k}, k=0,1$, are given in (2.17) above. By means of the tower property, we therefore obtain from (4.9) that

$$
\begin{aligned}
& E\left[e^{-\left(A_{T}-A_{t}\right)} C\left(Y_{T}\right) I\left(t<\theta_{1} \leq T<\theta_{2}\right) \mid \mathcal{F}_{t}\right] \\
& =P\left(t<\theta_{1} \mid \mathcal{F}_{t}\right) C_{1}^{1}\left(T-t, Y_{t}\right)=\left(1-\Pi_{t}^{1}\right) C_{1}^{1}\left(T-t, Y_{t}\right)
\end{aligned}
$$

holds, for all $0 \leq t \leq T$, where the function $C_{1}^{1}(T-t, y)$ is given in (4.10)-(4.11) above. 


\subsection{The third term}

Let us complete with computing the third term in (2.6). For this purpose, we observe that the equalities $Y_{s} / Y_{t}=Y_{s}^{2} / Y_{t}^{2}$ for $t \leq s \leq T$ and $A_{T}-A_{t}=Y_{t}\left(A_{T}^{2}-A_{t}^{2}\right) / Y_{t}^{2}$ hold on the event $\left\{\theta_{1}<\theta_{2} \leq t\right\}$, the equality $Y_{t}\left(Y_{\theta_{2}} / Y_{t}\right)\left(Y_{s} / Y_{\theta_{2}}\right)=Y_{t}\left(Y_{\theta_{2}}^{1} / Y_{t}^{1}\right)\left(Y_{s}^{2} / Y_{\theta_{2}}^{2}\right)$ holds on the event $\left\{\theta_{1} \leq t<\theta_{2} \leq s\right\}$ for $s \leq T$ and $A_{T}-A_{\theta_{2}}+A_{\theta_{2}}-A_{t}=Y_{t}\left(Y_{\theta_{2}}^{1} / Y_{t}^{1}\right)\left(A_{T}^{2}-A_{\theta_{2}}^{2}\right) / Y_{\theta_{2}}^{2}+$ $Y_{t}\left(A_{\theta_{2}}^{1}-A_{t}^{1}\right) / Y_{t}^{1}$ holds on $\left\{\theta_{1} \leq t<\theta_{2} \leq T\right\}$, while the equality $Y_{t}\left(Y_{\theta_{1}} / Y_{t}\right)\left(Y_{\theta_{2}} / Y_{\theta_{1}}\right)\left(Y_{s} / Y_{\theta_{2}}\right)=$ $Y_{t}^{0}\left(Y_{\theta_{1}}^{0} / Y_{t}^{0}\right)\left(Y_{\theta_{2}}^{1} / Y_{\theta_{1}}^{1}\right)\left(Y_{s}^{2} / Y_{\theta_{2}}^{2}\right)$ holds on the event $\left\{t<\theta_{1}<\theta_{2} \leq s\right\}$ for $s \leq T$ and $A_{T}-A_{\theta_{2}}+$ $A_{\theta_{2}}-A_{\theta_{1}}+A_{\theta_{1}}-A_{t}=Y_{t}^{0}\left(Y_{\theta_{1}}^{0} / Y_{t}^{0}\right)\left(Y_{\theta_{2}}^{1} / Y_{\theta_{1}}^{1}\right)\left(A_{T}^{2}-A_{\theta_{2}}^{2}\right) / Y_{\theta_{2}}^{2}+Y_{t}^{0}\left(Y_{\theta_{1}}^{0} / Y_{t}^{0}\right)\left(A_{\theta_{2}}^{1}-A_{\theta_{1}}^{1}\right) / Y_{\theta_{1}}^{1}+A_{\theta_{1}}^{0}-A_{t}^{0}$ holds on $\left\{t<\theta_{1}<\theta_{2} \leq T\right\}$, so that

$$
\begin{aligned}
& E\left[e^{-\left(A_{T}-A_{t}\right)} C\left(Y_{T}\right) I\left(\theta_{2} \leq T\right) \mid \mathcal{G}_{t}^{2}\right] \\
& =E\left[e^{-\left(A_{T}-A_{t}\right)} C\left(Y_{t}\left(Y_{T} / Y_{t}\right)\right) I\left(\theta_{1}<\theta_{2} \leq t\right) \mid \mathcal{G}_{t}^{2}\right] \\
& +E\left[e^{-\left(A_{T}-A_{\theta_{2}}\right)-\left(A_{\theta_{2}}-A_{t}\right)} C\left(Y_{t}\left(Y_{\theta_{2}} / Y_{t}\right)\left(Y_{T} / Y_{\theta_{2}}\right)\right) I\left(\theta_{1} \leq t<\theta_{2} \leq T\right) \mid \mathcal{G}_{t}^{2}\right] \\
& +E\left[e^{-\left(A_{T}-A_{\theta_{2}}\right)-\left(A_{\theta_{2}}-A_{\theta_{1}}\right)-\left(A_{\theta_{1}}-A_{t}\right)} C\left(Y_{t}\left(Y_{\theta_{1}} / Y_{t}\right)\left(Y_{\theta_{2}} / Y_{\theta_{1}}\right)\left(Y_{T} / Y_{\theta_{2}}\right)\right) I\left(t<\theta_{1}<\theta_{2} \leq T\right) \mid \mathcal{G}_{t}^{2}\right] \\
& =I\left(\theta_{1}<\theta_{2} \leq t\right) E\left[e^{-Y_{t}\left(A_{T}^{2}-A_{t}^{2}\right) / Y_{t}^{2}} C\left(Y_{t}\left(Y_{T}^{2} / Y_{t}^{2}\right)\right) \mid \mathcal{G}_{t}^{2}\right] \\
& +I\left(\theta_{1} \leq t<\theta_{2}\right) E\left[e^{-Y_{t}\left(Y_{\theta_{2}}^{1} / Y_{t}^{1}\right)\left(A_{T}^{2}-A_{\theta_{2}}^{2}\right) / Y_{\theta_{2}}^{2}-Y_{t}\left(A_{\theta_{2}}^{1}-A_{t}^{1}\right) / Y_{t}^{1}} C\left(Y_{t}\left(Y_{\theta_{2}}^{1} / Y_{t}^{1}\right)\left(Y_{T}^{2} / Y_{\theta_{2}}^{2}\right)\right) I\left(t<\theta_{2} \leq T\right) \mid \mathcal{G}_{t}^{2}\right] \\
& +I\left(t<\theta_{1}\right) E\left[e^{-Y_{t}^{0}\left(Y_{\theta_{1}}^{0} / Y_{t}^{0}\right)\left(Y_{\theta_{2}}^{1} / Y_{\theta_{1}}^{1}\right)\left(A_{T}^{2}-A_{\theta_{2}}^{2}\right) / Y_{\theta_{2}}^{2}-Y_{\theta_{1}}^{0}\left(A_{\theta_{2}}^{1}-A_{\theta_{1}}^{1}\right) / Y_{\theta_{1}}^{1}-\left(A_{\theta_{1}}^{0}-A_{t}^{0}\right)}\right. \\
& \left.\quad \times C\left(Y_{t}^{0}\left(Y_{\theta_{1}}^{0} / Y_{t}^{0}\right)\left(Y_{\theta_{2}}^{1} / Y_{\theta_{1}}^{1}\right)\left(Y_{T}^{2} / Y_{\theta_{2}}^{2}\right)\right) I\left(t<\theta_{1}<\theta_{2} \leq T\right) \mid \mathcal{G}_{t}^{2}\right]
\end{aligned}
$$

holds, for all $0 \leq t \leq T$. Firstly, taking into account the fact that $\mathcal{G}_{t}^{2}$ coincides with $\mathcal{F}_{t}^{2}$ on the event $\left\{\theta_{1}<\theta_{2} \leq t\right\}$, by virtue of the Markov property of the process $\left(Y^{2}, A^{2}\right)$, and the fact that the random variable $Y_{s}^{2} / Y_{t}^{2}$ has the same law as $Y_{s-t}^{2}$, for each $0 \leq s \leq t$, we get

$$
\begin{aligned}
& I\left(\theta_{1}<\theta_{2} \leq t\right) E\left[e^{-Y_{t}\left(A_{T}^{2}-A_{t}^{2}\right) / Y_{t}^{2}} C\left(Y_{t}\left(Y_{T}^{2} / Y_{t}^{2}\right)\right) \mid \mathcal{G}_{t}^{2}\right] \\
& =I\left(\theta_{1}<\theta_{2} \leq t\right) E\left[e^{-Y_{t}\left(A_{T}^{2}-A_{t}^{2}\right) / Y_{t}^{2}} C\left(Y_{t}\left(Y_{T}^{2} / Y_{t}^{2}\right)\right) \mid \mathcal{F}_{t}^{2}\right]=I\left(\theta_{1}<\theta_{2} \leq t\right) C_{2}^{0}\left(T-t, Y_{t}\right)
\end{aligned}
$$

where

$$
C_{2}^{0}(T-t, y)=E\left[e^{-y A_{T-t}^{2}} C\left(y\left(Y_{T}^{2} / Y_{t}^{2}\right)\right)\right]=\int_{0}^{\infty} \int_{0}^{\infty} e^{-y a} C(y z) g_{2}(T-t, z, a) d z d a
$$

and the function $g_{2}$ is given in (2.17) above. Hence, using the tower property, we obtain from (4.13) and (4.14) that

$$
\begin{aligned}
& E\left[e^{-\left(A_{T}-A_{t}\right)} C\left(Y_{T}\right) I\left(\theta_{1}<\theta_{2} \leq t\right) \mid \mathcal{F}_{t}\right] \\
& =P\left(\theta_{1}<\theta_{2} \leq t \mid \mathcal{F}_{t}\right) C_{2}^{0}\left(T-t, Y_{t}\right)=\Pi_{t}^{2} C_{2}^{0}\left(T-t, Y_{t}\right)
\end{aligned}
$$

holds, for all $0 \leq t \leq T$, where the function $C_{2}^{0}(T-t, y)$ is given in (4.15) above.

Secondly, taking into account the fact that $\mathcal{G}_{t}^{2}$ coincides with $\mathcal{F}_{t}^{2}$ on the event $\left\{\theta_{1} \leq t<\theta_{2}\right\}$, we can apply the generalised key lemma for the filtrations $\left(\mathcal{G}_{t}^{2}\right)_{t \geq 0}$ and $\left(\mathcal{F}_{t}^{1}\right)_{t \geq 0}$ on $\left\{\theta_{1} \leq t<\theta_{2}\right\}$ 
to get

$$
\begin{aligned}
& I\left(\theta_{1} \leq t<\theta_{2}\right) E\left[e^{-Y_{t}\left(Y_{\theta_{2}}^{1} / Y_{t}^{1}\right)\left(A_{T}^{2}-A_{\theta_{2}}^{2}\right) / Y_{\theta_{2}}^{2}-Y_{t}\left(A_{\theta_{2}}^{1}-A_{t}^{1}\right) / Y_{t}^{1}} C\left(Y_{t}\left(Y_{\theta_{2}}^{1} / Y_{t}^{1}\right)\left(Y_{T}^{2} / Y_{\theta_{2}}^{2}\right)\right) I\left(t<\theta_{2} \leq T\right) \mid \mathcal{G}_{t}^{2}\right] \\
& =I\left(\theta_{1} \leq t<\theta_{2}\right) \frac{E\left[e^{-Y_{t}\left(Y_{\theta_{2}}^{1} / Y_{t}^{1}\right)\left(A_{T}^{2}-A_{\theta_{2}}^{2}\right) / Y_{\theta_{2}}^{2}-Y_{t}\left(A_{\theta_{2}}^{1}-A_{t}^{1}\right) / Y_{t}^{1}} C\left(Y_{t}\left(Y_{\theta_{2}}^{1} / Y_{t}^{1}\right)\left(Y_{T}^{2} / Y_{\theta_{2}}^{2}\right)\right) I\left(t<\theta_{2} \leq T\right) \mid \mathcal{F}_{t}^{1}\right]}{P\left(\theta_{1} \leq t<\theta_{2} \mid \mathcal{F}_{t}^{1}\right)} \\
& =I\left(\theta_{1} \leq t<\theta_{2}\right) C_{2}^{1}\left(T-t, Y_{t}\right)
\end{aligned}
$$

for $0 \leq t \leq T$. Here, using the fact that the $\mathcal{F}_{t}^{1}$-measurable random variable $Y_{t}$ is independent of $\left(Y_{\theta_{2}}^{1} / Y_{t}^{1}\right)\left(Y_{T}^{2} / Y_{\theta_{2}}^{2}\right)$ on the event $\left\{\theta_{1} \leq t<\theta_{2} \leq T\right\}$, by virtue of the independence of the exponential random variables $\zeta_{1} \equiv \theta_{1}, \zeta_{2} \equiv \theta_{2}-\theta_{1}$, and $Y^{k}, k=1,2$, as well as the Markov property of the processes $\left(Y^{k}, A^{k}\right), k=1,2$, and the fact that the random variables $Y_{s}^{k} / Y_{t}^{k}$ have the same laws as $Y_{s-t}^{k}, k=1,2$, for each $0 \leq t \leq s$, we have

$$
\begin{aligned}
& C_{2}^{1}(T-t, y)=\frac{E\left[e^{-y A_{\zeta_{1}+\zeta_{2}-t}^{1}} \widehat{C}_{2}^{1}\left(T-\left(\zeta_{1}+\zeta_{2}\right), y Y_{\zeta_{1}+\zeta_{2}-t}^{1}\right) I\left(t<\zeta_{1}+\zeta_{2} \leq T\right) \mid \zeta_{1}\right]}{P\left(t<\zeta_{1}+\zeta_{2} \mid \zeta_{1}\right)} \\
& =\int_{t}^{T} \int_{0}^{\infty} \int_{0}^{\infty} e^{-y a} \widehat{C}_{2}^{1}(T-v, y z) \lambda e^{-\lambda(v-t)} g_{1}(v-t, z, a) d v d z d a
\end{aligned}
$$

with

$$
\widehat{C}_{2}^{1}(T-v, y)=E\left[e^{-y A_{T-v}^{2}} C\left(y Y_{T-v}^{2}\right)\right]=\int_{0}^{\infty} \int_{0}^{\infty} e^{-y a} C(y z) g_{2}(T-v, z, a) d z d a
$$

for $0 \leq t<v \leq T$, and the functions $g_{k}, k=1,2$, are given in (2.17) above. Note that the expression in (4.18) does not depend on $\zeta_{1}$, because of the exponential distribution of the random variable $\zeta_{2}$. Thus, by means of the tower property, we obtain from (4.17) that

$$
\begin{aligned}
& E\left[e^{-\left(A_{T}-A_{t}\right)} I\left(\theta_{1} \leq t<\theta_{2} \leq T\right) \mid \mathcal{F}_{t}\right] \\
& =P\left(\theta_{1} \leq t<\theta_{2} \mid \mathcal{F}_{t}\right) C_{2}^{1}\left(T-t, Y_{t}\right)=\left(\Pi_{t}^{1}-\Pi_{t}^{2}\right) C_{2}^{1}\left(T-t, Y_{t}\right)
\end{aligned}
$$

holds, for all $0 \leq t \leq T$, where the function $C_{2}^{1}(T-t, y)$ is given in (4.18)-(4.19) above.

Finally, taking into account the fact that $\mathcal{G}_{t}^{2}$ coincides with $\mathcal{F}_{t}^{0}$ on the event $\left\{t<\theta_{1}\right\}$, we apply the key lemma for the filtrations $\left(\mathcal{G}_{t}^{2}\right)_{t \geq 0}$ and $\left(\mathcal{F}_{t}^{0}\right)_{t \geq 0}$ to get

$$
\begin{aligned}
& I\left(t<\theta_{1}\right) E\left[e^{-Y_{t}^{0}\left(Y_{\theta_{1}}^{0} / Y_{t}^{0}\right)\left(Y_{\theta_{2}}^{1} / Y_{\theta_{1}}^{1}\right)\left(A_{T}^{2}-A_{\theta_{2}}^{2}\right) / Y_{\theta_{2}}^{2}-Y_{\theta_{1}}^{0}\left(A_{\theta_{2}}^{1}-A_{\theta_{1}}^{1}\right) / Y_{\theta_{1}}^{1}-\left(A_{\theta_{1}}^{0}-A_{t}^{0}\right)}\right. \\
& \left.\quad \times C\left(Y_{t}^{0}\left(Y_{\theta_{1}}^{0} / Y_{t}^{0}\right)\left(Y_{\theta_{2}}^{1} / Y_{\theta_{1}}^{1}\right)\left(Y_{T}^{2} / Y_{\theta_{2}}^{2}\right)\right) I\left(t<\theta_{1}<\theta_{2} \leq T\right) \mid \mathcal{G}_{t}^{2}\right] \\
& =\frac{I\left(t<\theta_{1}\right)}{P\left(t<\theta_{1} \mid \mathcal{F}_{t}^{0}\right)} E\left[e^{-Y_{t}^{0}\left(Y_{\theta_{1}}^{0} / Y_{t}^{0}\right)\left(Y_{\theta_{2}}^{1} / Y_{\theta_{1}}^{1}\right)\left(A_{T}^{2}-A_{\theta_{2}}^{2}\right) / Y_{\theta_{2}}^{2}-Y_{\theta_{1}}^{0}\left(A_{\theta_{2}}^{1}-A_{\theta_{1}}^{1}\right) / Y_{\theta_{1}}^{1}-\left(A_{\theta_{1}}^{0}-A_{t}^{0}\right)}\right. \\
& \left.\quad \times C\left(Y_{t}^{0}\left(Y_{\theta_{1}}^{0} / Y_{t}^{0}\right)\left(Y_{\theta_{2}}^{1} / Y_{\theta_{1}}^{1}\right)\left(Y_{T}^{2} / Y_{\theta_{2}}^{2}\right)\right) I\left(t<\theta_{1}<\theta_{2} \leq T\right) \mid \mathcal{F}_{t}^{0}\right] \\
& =I\left(t<\theta_{1}\right) C_{2}^{2}\left(T-t, Y_{t}\right)
\end{aligned}
$$

for all $0 \leq t \leq T$. Here, by virtue of the independence of $\zeta_{1} \equiv \theta_{1}, \zeta_{2} \equiv \theta_{2}-\theta_{1}$, and $Y^{k}$, $k=0,1,2$, as well as the Markov property of the processes $\left(Y^{k}, A^{k}\right), k=0,1,2$, and the fact 
that the random variables $Y_{s}^{k} / Y_{t}^{k}$ have the same laws as $Y_{s-t}^{k}, k=0,1,2$, for each $0 \leq t \leq s$, we have

$$
\begin{aligned}
& C_{2}^{2}(T-t, y)=E\left[e^{-y A_{\zeta_{1}-t}^{0}} \widetilde{C}_{2}^{2}\left(T-\zeta_{1}, y Y_{\zeta_{1}-t}^{0}\right) I\left(t<\zeta_{1} \leq T\right)\right] / P\left(t<\zeta_{1}\right) \\
& =\int_{t}^{T} \int_{0}^{\infty} \int_{0}^{\infty} e^{-y a} \widetilde{C}_{2}^{2}(T-u, y z) \lambda e^{-\lambda(u-t)} g_{0}(u-t, z, a) d u d z d a
\end{aligned}
$$

with

$$
\begin{aligned}
& \widetilde{C}_{2}^{2}(T-u, y)=E\left[e^{-y A_{\zeta_{2}}^{1}} \bar{C}_{2}^{2}\left(T-u-\zeta_{2}, y Y_{\zeta_{2}}^{1}\right) I\left(\zeta_{2} \leq T-u\right)\right] \\
& =\int_{u}^{T} \int_{0}^{\infty} \int_{0}^{\infty} e^{-y a} \bar{C}_{2}^{2}(T-v, y z) \lambda e^{-\lambda(v-u)} g_{1}(v-u, z, a) d v d z d a
\end{aligned}
$$

and

$$
\bar{C}_{2}^{2}(T-v, y)=E\left[e^{-y A_{T-v}^{2}} C\left(y Y_{T-v}^{2}\right)\right]=\int_{0}^{\infty} \int_{0}^{\infty} e^{-y a} C(y z) g_{2}(T-v, z, a) d z d a
$$

for $0 \leq t<u<v \leq T$, and the functions $g_{k}, k=0,1,2$, are given in (2.17) above. By means of the tower property, we therefore obtain from (4.5) and (4.21) that:

$$
\begin{aligned}
& E\left[e^{-\left(A_{T}-A_{t}\right)} C\left(Y_{T}\right) I\left(t<\theta_{1}<\theta_{2} \leq T\right) \mid \mathcal{F}_{t}\right] \\
& =P\left(t<\theta_{1} \mid \mathcal{F}_{t}\right) C_{2}^{2}\left(T-t, Y_{t}\right)=\left(1-\Pi_{t}^{1}\right) C_{2}^{2}\left(T-t, Y_{t}\right)
\end{aligned}
$$

holds, for all $0 \leq t \leq T$, where the function $C_{2}^{2}(T-t, y)$ is given in (4.22)-(4.24) above.

Therefore, summarising the facts proved above, we are now ready to formulate the following assertion.

Proposition 4.1. Suppose that $r=0$. The rational price of the European contingent claim in (2.5) and (2.6) under partial information contained in $\left(\mathcal{F}_{t}\right)_{t \geq 0}$ is given by the sum of the terms in (4.4), (4.8), (4.12), (4.16), (4.20) and (4.25).

Acknowledgments. The authors thank a Managing Editor for his useful comments, which helped to improve the presentation of the paper. This research benefited from the support of the 'Chaire Marchés en Mutation', French Banking Federation and ILB, Labex ANR 11-LABX0019. This research was supported by a Small Grant from the Suntory and Toyota International Centres for Economics and Related Disciplines (STICERD) at the London School of Economics and Political Science.

\section{References}

[1] Bielecki, T. R. and Rutkowski, M. (2004). Credit Risk: Modeling, Valuation and Hedging. (Second Edition) Springer, Berlin.

[2] Brody, D. C., Hughston, L. P. and Macrina, A. (2008). Information-based asset pricing. International Journal of Theoretical and Applied Finance 11 (107-142). 
[3] Dellacherie, C. (1972). Capacités et processus stochastiques. Springer, Berlin.

[4] Dufresne, D. (2001). The integral of geometric Brownian motion. Advances in Applied Probability 33 (223-241).

[5] Elliott, R. J., Aggoun, L. and Moore, J. B. (1995). Hidden Markov Models: Estimation and Control. Springer, New York.

[6] Gapeev, P. V. and Jeanblanc, M. (2009). Pricing of contingent claims in a twodimensional model with random dividends. International Journal of Theoretical and Applied Finance 12 (1091-1104).

[7] Gapeev, P. V. and Jeanblanc, M. (2010). Pricing and filtering in a twodimensional switching model with partial information. International Journal of Theoretical and Applied Finance 13 (1001-1017).

[8] Gapeev, P. V. and Peskir, G. (2006). The Wiener disorder problem with finite horizon. Stochastic Processes and their Applications 116(12) (1770-1791).

[9] Kallianpur, G. (1980). Stochastic filtering theory. Springer, New York.

[10] Liptser, R. S. and Shiryaev, A. N. (2001). Statistics of Random Processes I. (Second Edition, First Edition 1977) Springer, Berlin.

[11] Revuz, D. and Yor, M. (1999). Continuous Martingales and Brownian Motion. (Third Edition) Springer, Berlin.

[12] Schröder, M. (2003). On the integral of geometric Brownian motion. Advances in Applied Probability 35 (159-183).

[13] Shiryaev, A. N. (1965). Some exact formulas in a 'disorder' problem. Theory of Probability and its Applications 10 (348-354).

[14] Shiryaev, A. N. (1973). Statistical Sequential Analysis. American Mathematical Society, Providence.

[15] Shiryaev, A. N. (1978). Optimal Stopping Rules. Springer, Berlin.

[16] Yor, M. (1992). On some exponential functionals of Brownian motion. Advances in Applied Probability 24 (509-531). 